\title{
Aproveitamento de Água da Chuva: um Dimensionamento para o Caso de Aviários
}

\author{
Rainwater Use: A Sizing for the Case of Poultry Houses
}

\author{
Genilso Gomes de Proença | Carla Adriana Pizarro Schmidt
}

Data de entrada: 26/07/2013 | Data de aprovação: 24/02/2014

DOI: http://dx.doi.org/10.4322/dae.2014.125

\section{Resumo}

O objetivo do estudo foi o de apresentar o dimensionamento de uma cisterna, para coleta de água da chuva, nas condições climáticas do município de Medianeira - PR, o qual apresenta valores entre 90 e $275 \mathrm{~mm}$ de chuva por mês. No experimento foram utilizadas equações disponibilizadas pela EMBRAPA para o cálculo e o estudo foi realizado focando um aviário de $1000 \mathrm{~m}^{2}$, que para a região apresentaria um potencial de captação médio de $146 \mathrm{~m}^{3}$ de água por mês. Observou-se que a quantidade de água que seria coletada, mostrou-se suficiente para manter a demanda das 12 mil aves alojadas no aviário em seu período de maior exigência de água sendo até suficiente para gerar excedentes por meio da instalação de uma cisterna de $60 \mathrm{~m}^{3}$.

Palavras-chave: Água de Chuva, Dimensionamento de Cisterna, Aviários.

\begin{abstract}
The study aim sizing a rainwater cistern, in climatic conditions of Medianeira - $P R$, which has monthly rains values between 90 and $275 \mathrm{~mm}$. Equations used in the accounts experiment were provided by EMBRAPA, this study was focused on a $1000 \mathrm{~m}^{2}$ poultry house, which present average capturing potential to this region of $146 \mathrm{~m}^{3}$ water per month. It was observed that the water amount that would be collected was sufficient to maintain the 12.000 birds housed in the poultry demand in its greater demand period for water being sufficient to generate excess amounts by installing a $60 \mathrm{~m}^{3}$ cistern.
\end{abstract}

Key-words: Rainfall, Cistern Sizing, Poultry Houses.

Genilso Gomes de Proença

Técnico Agrícola. Bacharel em Sistemas de Informação pela UNIGUAÇU. Especialista em Gestão Ambiental em Municípios pela UTFPR. Discente do Curso de Tecnólogo em Agronegócios da UNICESUMAR.

\section{Carla Adriana Pizarro Schmidt*}

Engenheira Agrônoma e Doutora em Agronomia pela UEL. Docente Efetiva do Ensino Básico Técnico e Tecnológico da Universidade Tecnológica Federal do Paraná (UTFPR).

* Endereço para correspondências : UTFPR CÂMPUS MEDIANEIRA. Avenida Brasil, 4232. CEP 85884-000 - Caixa Postal 271 - Medianeira - PR - Brasil. Telefone Geral +55 (45) 3240-8000 - Fax : +55 (45) 3240-8101.

e-mail: carlaschmidt@utfpr.edu.br 


\section{Introdução}

A captação de água da chuva e seu posterior armazenamento em cisternas para consumo é uma prática milenar em várias regiões do mundo. Atualmente tal tecnologia vem se modernizando por meio da incorporação de novos conceitos e técnicas construtivas, bem como a preocupação com a segurança sanitária (ANDRADE NETO, 2004).

Toda água utilizada no aviário, independente de ser de chuva ou poço, estar ou não contaminada, por coliformes ou outros microrganismos, deve receber pelos menos dois tratamentos básicos antes de chegar aos frangos: Filtração através de "filtro de areia lento" e desinfecção química. A indicação de se tratar a água independe da contaminação, decorre do fato que, quando se detecta a contaminação, a água já foi ingerida pelos frangos e o tratamento tem função preventiva (MACEDO, 2005).

A aplicação dos tratamentos à água é muito importante, o estudo de Xavier et al. (2011) alerta ao fato de que 90\% das amostras de água da chuva armazenadas por comunidades rurais em cisternas localizadas no interior do estado de Pernambuco estavam com níveis elevados de contaminação microbiológica, sendo portanto consideradas impróprias para o consumo.

Jaenisch (2003), recomenda para o tratamento da água de dessedentação, uma cloração pela adição de 3 (três) ppm de Cloro (hipoclorito de sódio), mas ressalta, que a água usada para vacinações das aves não pode ser clorada. Já Macedo (2005), recomenda uma dosagem superior de 5 (cinco) ppm de Cloro, pois segundo ele a OMS (Organização Mundial de Saúde) considera que uma concentração de 0,5 mg/L de cloro livre residual na água, depois de um tempo de contato de 30 minutos, garante uma desinfecção satisfatória da água.

Para melhor conservação da água armazenada na cisterna, recomenda-se que esta seja enterrada, pois assim a temperatura da água fica mais estável e reduz a proliferação de microrganismos. É importante ainda que as cisternas permaneçam sempre fechadas, evitando-se a entrada de insetos, roedores, pássaros e sujeiras (OLIVEIRA; GIRON; SCHIMIDT, 2009).

Existem diversos modelos de cisterna para armazenagem de água da chuva. Os materiais mais utilizados de acordo com Oliveira, Giron e Schimidt (2009), na construção são: vinimanta de PVC, manta de PEAD, fibra de vidro, alvenaria, ferro cimento ou concreto armado. Os reservatórios em fibra de vidro e alvenaria são mais empregados para meno- res volumes de armazenagem, e os reservatórios de PVC, PEAD e concreto armado são recomendados para armazenagem de grandes volumes.

Gnadlinger (2005), do Instituto Regional da Pequena Agropecuária Apropriada, apresenta em seu estudo diferentes tipos de cisternas de baixo custo que podem ser construídas em comunidades rurais com vistas ao armazenamento e fornecimento dessa água aos animais, a saber, cisternas de placas de cimento, de tela-cimento, de tijolos, de ferro cimento, de cal e de plástico, demonstrando que naquela época os custos de implantação de cada $10 \mathrm{~m}^{3}$ dessas cisternas descritas giravam entre $\mathrm{R} \$ 175,43$ e $\mathrm{R} \$ 322,50$, o autor apresenta também informações que permitem observar que a construção de cisternas maiores tende a baratear um pouco esses valores.

Já Brito et al. (2005), apresentam um cálculo de custo bem superior de $\mathrm{R} \$ 2.060,83$, para a construção de uma cisterna de placas pré-moldadas com capacidade para armazenar $16 \mathrm{~m}^{3}$ de água, mas apresentam adicionados aos seus cálculos o custo do sistema de filtragem e também de bebedouro. Os autores explicam que em casos de locais com possibilidade de uso de força da gravidade para deslocar a água tais custos calculados poderiam ser reduzidos.

No resultados do estudo de Zanin et al. (2009), observa-se que o retorno do investimento em aproveitamento da água da chuva é atrativo e ocorre num período entre 3 e 4 anos. No entanto, os autores destacam que o ganho maior é para o meio ambiente, pois a utilização de cisternas pode facilitar o aproveitamento da água das chuvas.

Com vistas à busca de alternativas ambientalmente mais sustentáveis na cadeia de produção de Aves, o objetivo do presente trabalho foi o de apresentar um exemplo de dimensionamento de cisterna, específico para as condições ambientais do município de Medianeira-PR exemplificando os cálculos com a situação de um Aviário.

\section{Material E Métodos}

Os dados de precipitação média foram obtidos do banco de dados da Cooperativa Agroindustrial Lar e referem-se ao controle de pluviosidade média de 20 anos do município de Medianeira, localizado no oeste do estado do Paraná.

A metodologia de cálculo de tamanho de cisternas utilizada no presente estudo encontra-se descrita pela EMBRAPA Suínos e Aves (GRINGS; OLIVEIRA, 2005) e pressupõe o emprego das Equações 1 e 2. 


$$
V_{c}=\left(V_{d} \times N_{d i a}\right)+10 \%
$$

Equação (1)

\section{Sendo:}

$V_{c}=$ Volume da cisterna $\left(\mathrm{m}^{3}\right)$

$V_{d}=$ Volume de demanda da água diária $\left(m^{3}\right)$

$N_{\text {dia }}=$ Número de dias de armazenagem

$10 \%$ = Acréscimo de $10 \%$ em função da evaporação no período de armazenagem considerado.

Considerando que uma chuva de $1 \mathrm{~mm}$ sobre uma área de $1 \mathrm{~m}^{2}$ produz 1 litro de água, pode-se calcular a fim de atender a necessidade calculada pela Equação 1 a área de telhado necessária para suprir a demanda por meio da Equação 2.

$$
A=V_{c} \div \text { Prec_Período }
$$

Equação (2)

\section{Sendo:}

$A=$ Área de telhado para captação $\left(m^{2}\right)$

$V c=$ Volume da cisterna $\left(\mathrm{m}^{3}\right)$

Prec_Período= precipitação media no período considerado para captação (m)

Também é possível determinar a quantidade de água disponível para captação no município com base nos dados pluviométricos médios dos últimos 20 anos e da área em projeção do telhado (Equação 3). Nesse caso necessita-se também do coeficiente de escoamento superficial do tipo de telhado também denominado coeficiente de runoff (Tabela 1).

$$
V_{m}=P x A \times C
$$

Equação (3)
Onde:

$V m=$ Volume médio mensal de água $(L)$;

$P=$ Precipitação média mensal (mm);

$A=$ Área de coleta em projeção $\left(m^{2}\right)$;

$C=$ Coeficiente de Runoff (Tabela 1).

Conforme Tomaz (2003), o coeficiente de runoff para diferentes tipos de telhas pode ser observado na Tabela 1.

\begin{tabular}{l|c}
\multicolumn{1}{c|}{ Material } & Coeficiente de Runoff \\
\hline Telhas cerâmicas & 0,8 a 0,9 \\
\hline Telhas esmaltadas & 0,9 a 0,95 \\
\hline Telhas corrugadas de metal & 0,8 a 0,9 \\
\hline Cimento amianto & 0,8 a 0,9 \\
\hline Plástico e PVC & 0,9 a 0,95
\end{tabular}

Fonte: Tomaz (2003)

Tabela 1 - Coeficientes de Runoff médios para diferentes tipos de telha

\section{Resultados E Discussão}

Assim, avaliando-se uma situação de grande demanda de água, de acordo com a Tabela 2, em um aviário com 12.000 frangos de 8 semanas, que necessitasse armazenar água por um período de 30 dias, o reservatório deveria ter capacidade para um volume mínimo de $102.960 \mathrm{~L}$ ou 102,96 m³.

Porém segundo Grings e Oliveira (2005), deve-se utilizar para o dimensionamento das cisternas a demanda de apenas 15 dias, dessa forma pode-se prever o tamanho recomendado para a cisterna por meio da Equação 1. Por meio desse cálculo, dever-se-ia ter para essa situação uma cisterna com capacidade para $56,63 \mathrm{~m}^{3}$.

Vale aqui destacar que, em alguns momentos onde a estiagem for superior a 15 dias, evento raro, porém possível para a região, o agricultor necessitaria fazer uso de outra fonte de abastecimento de água.

Levando-se em conta a demanda adicional de água utilizada na limpeza do aviário, 7 vezes por ano, disponibilizada na Tabela 2, que é de $10 \mathrm{~m}^{3}$ por lote sugere-se um aumento do volume da cisterna em aproximadamente $3,1 \mathrm{~m}^{3}$ de onde se pode chegar ao valor de $59,73 \mathrm{~m}^{3}$, ou seja, aproximadamente $60 \mathrm{~m}^{3}$.

Dessa forma recomenda-se nesse trabalho que seja adotada uma Cisterna de $60 \mathrm{~m}^{3}$ com a finalidade de suprir toda a demanda de água do aviário estudado. 


\begin{tabular}{c|c|c} 
Semana & $\mathrm{mL} /$ dia/Frango & L/dia/12 mil Frangos \\
\hline 1 & 32 & 384 \\
\hline 2 & 69 & 828 \\
\hline 3 & 104 & 1248 \\
\hline 4 & 143 & 1716 \\
\hline 5 & 179 & 2149 \\
\hline 6 & 214 & 2568 \\
\hline 7 & 250 & 3000 \\
\hline 8 & 286 & 3432 \\
\hline
\end{tabular}

Fonte: Girotto e Avila (2003)

Tabela 2 - Estimativa de consumo diário de água para aves

Considerando-se que de acordo com Girotto e Avila (2003), é necessário $1 \mathrm{~m}^{2}$ de espaço para criação de 12 frangos e o referido dimensionamento está sendo realizado para um lote de 12.000 frangos, o aviário deve possuir 1000 metros quadrados de telhado. Sabendo-se também que o volume de precipitação no município de Medianeira se situa geralmente entre 90 e 275 mm de chuva por mês, calculando-se com base na Equação 2 a área necessária para o mês de menor precipitação, $90 \mathrm{~mm}$ ou 0,090 m de chuva, o resultado de área necessária de telhado a ser direcionado para cisterna seria de $666 \mathrm{~m}^{2}$.

Observando-se também que o aviário todo tem $1000 \mathrm{~m}^{2}$ e que cada $\mathrm{mm}$ de chuva por $\mathrm{m}^{2}$ representa 1 litro de água, se pode também calcular pela Equação 3, supondo um runoff de 0,8, para saber quanto de água poderia ser coletada utilizando-se o tamanho total do aviário. Com esse raciocínio seria obtido um resultado de $72 \mathrm{~m}^{3}$ nos meses de menor precipitação e $220 \mathrm{~m}^{3}$ nos meses de maiores precipitações resultando em uma média de $146 \mathrm{~m}^{3}$ de água por mês. O que seria mais do que o suficiente para manter a cisterna, de aproximadamente $60 \mathrm{~m}^{3}$ calculada com base na demanda de água para dessedentação das aves do aviário, cheia na maior parte do ano.
ILKIU (2009), estudando a coleta de água da chuva para o caso de um aviário com capacidade para alojar 18 mil frangos, localizado no município de São Jorge do Oeste - PR, onde o volume de precipitação médio mensal se situa entre 97,4 e 236,1 mm chegou à necessidade de implantação de uma cisterna de $55 \mathrm{~m}^{3}$, por meio de cálculos realizados com base no método prático brasileiro. $\mathrm{O}$ valor por ele encontrado para a cisterna foi inferior, porém próximo ao encontrado no presente estudo, vale aqui lembrar que Medianeira possui volume médio de precipitação mensal também inferior ao apresentado pelo autor para São Jorge do Oeste.

O custo da água distribuída pela Sanepar para Comercial/Industrial, que atende a região avaliada nesse estudo é de $\mathrm{R} \$ 39,73$ para os primeiros $10 \mathrm{~m}^{3}$ mensais sendo que para cada $\mathrm{m}^{3}$ adicional é acrescido no valor da tarifa $\mathrm{R} \$ 4,47$ (SANEPAR, 2012). Por ser a demanda de água para a atividade muito superior aos $10 \mathrm{~m}^{3}$ o custo mensal de água, se utilizado na propriedade esse sistema de abastecimento, estaria próximo ao valor de $\mathrm{R} \$ 500,00$. Muitas propriedades da região possuem poços artesianos, mas mesmo assim deve-se considerar o custo da energia elétrica utilizada para o bombeamento da água até a superfície, valendo lembrar também que o tratamento químico também precisará ser realizado no

\begin{tabular}{|c|c|c|} 
Quantidade de frangos no lote & Número de lotes por ano & $\mathrm{m}^{3}$ de água/lote para higiene dos equipamentos \\
\hline 12000 & 7 & 10
\end{tabular}

Fonte: Bellaver e Oliveira, (2009)

Tabela 3 - Quantidade de água utilizada para limpeza de equipamento em granja de frangos 
caso do uso das águas subterrâneas na dessedentação das aves.

O tratamento da água por meio de filtragem e cloração é essencial tendo em vista que tanto a água pluvial como as subterrâneas trazem consigo muitas impurezas dissolvidas e se utilizadas sem o devido tratamento podem ocasionar inúmeras doenças, principalmente em aves que são animais muito sensíveis.

\section{Conclusão}

Com base nos cálculos realizados, para as condições do município de Medianeira-PR, para uma granja com 12.000 aves dispostas em um aviário de $1000 \mathrm{~m}^{2}$ deve-se construir uma cisterna com capacidade de armazenamento de $60 \mathrm{~m}^{3}$, e poder-se-ia utilizar a coleta de água de apenas $666 \mathrm{~m}^{2}$ deste telhado sendo que tal cisterna supriria a necessidade de água para dessedentação das aves e também para as atividades de higienização necessárias por um período calculado de 15 dias.

O período de 15 dias, na maioria dos meses é o intervalo máximo de dias sem ocorrência de chuva para região estudada, em casos extremos o agricultor precisará lançar mão de um abastecimento alternativo que não deve ser completamente descartado.

Os custos de implantação dependerão do tipo de cisterna escolhida para cada caso específico bem como do tipo e tamanho dos equipamentos de desvio de fluxo das primeiras águas, de filtração e cloração de água, indispensáveis para o uso do recurso com vistas à segurança alimentar dos animais.

\section{Referências}

ANDRADE NETO, C O de. Proteção Sanitária das Cisternas Rurais. In: XI SIMPÓSIO LUSO-BRASILEIRO DE ENGENHARIA SANITÁRIA E AMBIENTAL. 2004, Natal, Brasil. Anais ... Natal: ABES/APESB/APRH. 2004.

BELLAVER, C, OLIVEIRA, P.A. Balanço da água nas cadeias de aves e suínos. Avicultura Industrial, n.10, p.39-44, 2009.

BRITO, L.T.L.; PORTO, E.R.; SILVA, A.S.; CAVALCANTI, N.B. Cisterna rural: Água para o Consumo Animal. IEH - Instituto de Estudios del Hambre. 2005.

GIROTTO, A.F.; AVILA, V.S. Sistema de Produção de Frangos de Corte. EMBRAPA Suínos e Aves. Versão Eletrônica. Jan., 2003. ISSN 1678-8850.

GNADLINGER, J. Apresentação Técnica de Dife- rentes Tipos de Cisternas, Construídas em Comunidades Rurais do Semi-árido Brasileiro. IRPAA - Instituto Regional da Pequena Agropecuária Apropriada. 2005.

GRINGS, V.H.; OLIVEIRA, P.A.V. Cisternas para Armazenagem de água da Chuva. MAPA - Ministério da Agricultura e Pecuária e EMBRAPA Suínos e Aves, 2005.

ILKIU, R.V. Aproveitamento de água pluvial em aviários. Trabalho de Diplomação (Graduação em Engenharia Ambiental). Faculdade Dinâmica das Cataratas - UDC. 2009. 58p.

JAENISCH, F. R. F. Produção Frangos de Corte. Sistema de Produção, 2. Versão Eletrônica. Jul., 2003. ISSN 1678-8850. Embrapa Suínos e Aves.

MACEDO, J.A.B. Processo de desinfecção de água utilizada na avicultura por derivados clorados, como barreira sanitária ao vírus Influenza aviária. Faculdades Integradas Viana Júnior. Universidade Federal de Juiz de Fora, 2005.

OLIVEIRA, P.A.V.; GIRON, J.; SCHIMIDT, A. Cisterna para captação e armazenamento de água da chuva. Dia de Campo - Suinocultura e Meio Ambiente. Concórdia: EMBRAPA Suínos e Aves, 07 e 08 out. 2009.

SANEPAR. Tabela de tarifas de saneamento básico. Contas vencíveis a partir de 21 de março de 2012.

TOMAZ, P. Aproveitamento de água de chuva. São Paulo: Navegar, 2003, 180p.

XAVIER, R.P. et al. Microbiological quality of drinking rainwater in the inland region of Pajeú, Pernambuco, Northeast Brazil. Revista do Instituto de Medicina Tropical de São Paulo, São Paulo, v. 53, n. 3, June 2011.

ZANIN, A.; BALDISSERA, A.L.; BAGATINI, F.M.; BENDER, S.L. Aproveitamento sustentável da água da chuva em propriedades rurais: o uso de cisternas. In: VI Simpósio de Excelência em Gestão e Tecnologia - VI SEGeT, 2009, Resende, Brasil. Anais ... Resende: AEDB - Associação Educacional Dom Bosco. 2009. 


\section{O site da Revista DAE está repleto de novidades}

Assine gratuitamente o boletim eletrônico, é só se cadastrar!

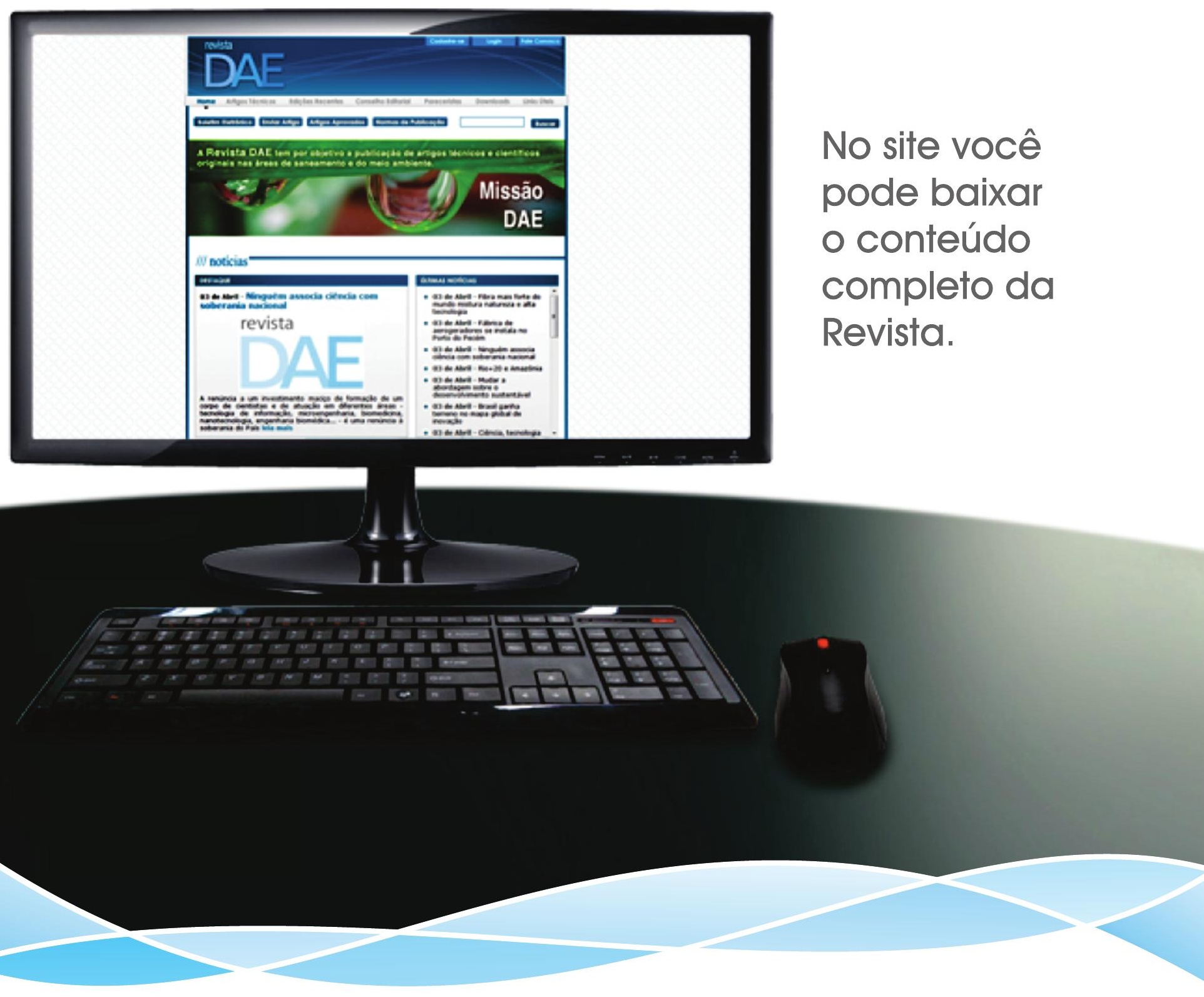

